\title{
OBSTRUCTION THEORY WITH COEFFICIENTS IN A SPECTRUM $\left({ }^{1}\right)$
}

\author{
BY \\ MARK MAHOWALD AND ROBERT RIGDON
}

\begin{abstract}
In this paper an obstruction theory with coefficients in a spectrum is developed. An idea of orientability of a fiber bundle with respect to a spectrum is introduced and for bundles orientable with respect to the spectrum a resolution is produced which corresponds to a modified Postnikov tower in the classical case.
\end{abstract}

1. Introduction. If $E=\left\{E_{n}\right\}$ is a spectrum with unit ( $\$ 2$ for definition) then it is possible to construct Adams type spectral sequences useful for studying $\pi_{*}(X)$ and $\{Y, X\}$. Our main purpose here is to describe the theory necessary to get analogous results for the cross-section problem with coefficients in the homology of the spectrum $E$. In $\$ 2$ we construct a well-known unstable resolution of the space $X$, and then use it to define the Adams spectral sequence converging to a quotient of $\{Y, X\}$ * (essentially the spectral sequence of $[1, \S 15])$. When the theory is applied to fibrations a question of stability becomes crucial and one way to handle it is to restrict attention to a fixed range of dimension. There is always a problem of inductively losing a dimension and the purpose of $\S 3$ is to set up the machinery necessary to avoid this problem.

In $\S 4$ the theory is applied to fibrations, and a spectral sequence of Adams type for enumerating lifting is defined. The approach to resolutions in this section is that of Anderson in [2] (except that Anderson works semisimplicially while we do not). One of the advantages of this approach which generalizes the work of McClendon [12] and Meyer [15] is that the enumeration problem is easier to handle than the lifting problem and, indeed, the lifting problem is reduced to an enumeration problem. This is accomplished by introducing Larmore's single obstruction [9] and is discussed in $§ 6$.

$\$ 7$ contains the main result of the paper which we hope will make the work useful. We define a notion of orientability with respect to $E$ for a fibration (definition 7.1). The condition is quite natural and seems to be 
capable of being checked for particular interesting examples. The key results are 7.2 and 7.3 which assert that orientability of a fibration implies orientability of the fibrations induced by the resolution. For the ordinary cohomology case of [10] this result was proved by Gitler and Mahowald [7]. The argument here even for the special case is simpler once the slightly more general machine is established.

2. The resolution $I(X)$. First some preliminaries. All spaces are assumed to have basepoints and all maps are assumed to be basepoint preserving. Throughout this paper, a spectrum $E=\left\{E_{m}\right\}$ with maps $\epsilon_{m}: S\left(E_{m-1}\right)=E_{m-1} \wedge S^{1}$ $\rightarrow E_{m}$ is assumed to satisfy the following conditions:

(a) Each $E_{m}$ is a pointed CW complex and $\epsilon_{m}: S\left(E_{m-1}\right) \rightarrow E_{m}$ embeds $S\left(E_{m-1}\right)$ as a subcomplex of $E_{m}$.

(b) Each $E_{m}$ is $(m-1)$-connected.

(c) $\pi_{0}(E)$ is cyclic.

(d) For all $t$, the map $\epsilon_{m}$ is a $(t+m)$-equivalence for all but finitely many $m$.

(e) There is given a map $\left\{l_{m}\right\}_{m} \geqslant_{m_{0}}: S \rightarrow E$ of spectra where $S$ is the sphere spectrum, such that $\iota_{m}=S\left(l_{m-1}\right)$ and $\left\{l_{m}\right\}_{*}: \pi_{0}(S) \rightarrow \pi_{0}(E)$ is an epimorphism.

A map $\left\{\alpha_{m}\right\}: E \rightarrow E^{\prime}$ of spectra is a collection of maps $\alpha_{m}: E_{m} \rightarrow$ $E_{m}^{\prime}$ defined for all but finitely many $m$, satisfying $\alpha_{m} \mid S\left(E_{m-1}\right)=S\left(\alpha_{m-1}\right)$ and $\alpha_{m} \iota_{m}=i_{m}^{\prime}$.

For any group $\pi, K(\pi)$ will denote a spectrum satisfying $\pi_{0}(K(\pi)) \approx$ $\pi, \pi_{i}(K(\pi))=0$ for $i>0$.

Let $X$ be a pointed space having the homotopy type of a pointed CW complex. Assume $X$ is simply-connected and $\pi_{*}(X)$ is of finite type.

The adjoint of $1 \wedge \epsilon_{m}: X \wedge E_{m-1} \wedge S^{1} \rightarrow X \wedge E_{m}$ is an embedding $X \wedge E_{m-1} \rightarrow \Omega\left(X \wedge E_{m}\right)$ and this induces an embedding $\Omega^{m-1}\left(X \wedge E_{m-1}\right)$ $\rightarrow \Omega^{m-1} \Omega\left(X \wedge E_{m}\right)=\Omega^{m}\left(X \wedge E_{m}\right)$.

Let $E(X)=\lim _{m \rightarrow \infty} \Omega^{m}\left(X \wedge E_{m}\right)$ where the limit is the direct limit of topological spaces. There is a natural inclusion $\iota_{X}: X \rightarrow E(X)$ which is the adjoint of $1 \wedge \iota_{m}: X \wedge S^{m} \rightarrow X \wedge E_{m}$ for each $m$. Because of the assumptions (b) and (d), for any $t$, the inclusion $\Omega^{m}\left(X \wedge E_{m}\right) \rightarrow E(X)$ is a $t$-equivalence for sufficiently large $m$. Clearly, $E(X)$ is functorial in both $X$ and $E$. $\pi_{*}(E(X))$ is $\widetilde{E}_{*}(X)$, the reduced $E$-homology of $X$, and $\left(\iota_{X}\right)_{*}: \pi_{*}(X)$ $\rightarrow \widetilde{E}_{*}(X)$ is the Hurewicz map.

There are natural maps

$$
E(\Omega(X)) \rightarrow \Omega(E(X))
$$

which induce isomorphisms of homotopy groups in dimensions $\leqslant 2 n$ if $X$ is 
n-connected, and

$$
E(X) \rightarrow \Omega(E(S(X)))
$$

which is always a weak homotopy equivalence. (2.1) is induced by the obvious map $\Omega(X) \wedge E_{m} \rightarrow \Omega\left(X \wedge E_{m}\right)$ and (2.2) is the composite

$$
E(X) \rightarrow E(\Omega(S(X))) \rightarrow \Omega(E(S(X))) .
$$

The resolution $I(X)$ of $X$ with respect to the spectrum $E$ is the following tower of principal fibrations over $X$.

$I(X)$

$$
X_{2} \stackrel{\iota}{\rightarrow} E\left(X_{2}\right)
$$

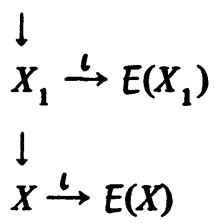

The map $X_{i+1} \rightarrow X_{i}$ is the principal fibration induced by the natural inclusion $X_{i} \stackrel{\iota}{\rightarrow} E\left(X_{i}\right)$. Diagram $I(X)$ is functorial in both $X$ and $E$.

The homotopy spectral sequence $\left\{U E_{r}(X ; E)\right\}$ of the tower $I(X)$ is an unstable spectral sequence with coefficients in $E$, indexed as follows:

$$
\begin{aligned}
U E_{1}^{s, t}(X ; E) & =\pi_{t-s}\left(E\left(X_{s}\right)\right), & & t-s \geqslant 0, \\
& =0, & & t-s \leqslant 0 .
\end{aligned}
$$

When $E=K(R)$, where $R$ is a ring, this is just the derived spectral sequence.

Convergence is as follows:

If $\pi_{0}(E) \approx \mathbf{Z},\left\{U E_{r}(X ; E)\right\} \Rightarrow \pi_{*}(X)$.

If $\pi_{0}(E) \approx \mathbf{Z}_{p}$, where $p$ is prime, $\left\{U E_{r}(X ; E)\right\} \Rightarrow C_{p} \pi_{*}(X)$. Here $C_{p}$ is the Serre class consisting of the abelian groups in which each nonzero element has finite order prime to $p$.

The precise meanings of $\Rightarrow$ and $\Rightarrow c_{p}$ are as follows: Set

$$
F^{s} \pi_{q}(X)=\operatorname{im}\left(\pi_{q}(X) \rightarrow \pi_{q}(X)\right), \quad F^{\infty} \pi_{q}(X)=\bigcap_{s} F^{s} \pi_{q}(X) .
$$

If $\pi_{0}(E) \approx \mathbf{Z}$, then $F^{\infty} \pi_{q}(X)=0$ and there are natural isomorphisms

$$
\tau^{s, t}:\left(F^{s} / F^{s+1}\right) \pi_{t-s}(X) \rightarrow U E_{\infty}^{s, t}(X ; E) .
$$


If $\pi_{0}(E) \approx \mathbf{Z}_{p}$, then $F^{\infty} \pi_{q}(X)$ is the subgroup of $\pi_{q}(X)$ generated by the elements having finite order prime to $p$, and there are natural $C_{p}$-isomorphisms

$$
\tau^{s, t}:\left(F^{s} / F^{s+1}\right) \pi_{t-s}(X) \rightarrow U E_{\infty}^{s, t}(X ; E) .
$$

Of course, $\tau^{s, t}$ is an isomorphism if $p U E_{\infty}^{s, t}(X ; E)=0$, which is always the case if $E$ is a ring spectrum.

Proof of convergence when $\pi_{0}(E) \approx Z$.

Lemma 2.4. Assume $\pi_{0}(E) \approx \mathrm{Z}$ and $H_{1}(E)=0$. If $X$ is n-connected, then $X_{i}$ is $(n+i)$-connected.

The proof of the lemma is by induction on $i$. By the exact homotopy sequence of the fibration $X_{i} \rightarrow X_{i-1} \stackrel{\iota}{\rightarrow} E\left(X_{i-1}\right)$, it suffices to show that $X_{i-1} \stackrel{\iota}{\rightarrow} E\left(X_{i-1}\right)$ is an $(n+i+1)$-equivalence. But for large $m, 1 \wedge^{\iota}$ : $X_{i-1} \wedge S^{m} \rightarrow X_{i-1} \wedge E_{m}$ is an $(n+i+m+1)$-equivalence by the inductive hypothesis since $\iota_{m}: S^{m} \rightarrow E_{m}$ is an $(m+1)$-equivalence. Taking the adjoint and passing to the limit yields the result.

By the lemma, $\left\{U E_{r}(X ; E)\right\} \Rightarrow \pi_{*}(X)$ if $E=K(\mathbf{Z})$. If $\pi_{0}(E) \approx \mathbf{Z}$, convergence follows from convergence for $E=K(Z)$ and the map of towers induced by the natural map $E \rightarrow K(\mathrm{Z})$ of spectra.

Proof of convergence when $\pi_{0}(E) \approx \mathbf{Z}_{p}$. Convergence when $E=K\left(Z_{p}\right)$ is well known. If $\pi_{0}(E) \approx \mathbf{Z}_{p}$, the map of towers induced by the map $E \rightarrow$ $K\left(\mathbf{Z}_{p}\right)$ establishes that every nonzero element in $F^{\infty} \pi_{*}(X)$ has finite order prime to $p$. That every element having order prime to $p$ is in $F^{\infty} \pi_{*}(X)$ follows from the fact that the homotopy class of $1 \wedge \iota_{m}: X_{i} \wedge S^{m} \rightarrow X_{i} \wedge E_{m}$ has order $p$ or is 0 . (Recall that the natural inclusion $X_{i} \rightarrow E\left(X_{i}\right)$ is the adjoint of $1 \wedge \iota_{m}$.) The existence of the $C_{p}$-isomorphism $\tau^{s, t}$ is readily verified.

Let $\Omega(I(X))$ be the tower of principal fibrations obtained by applying the loop functor to $I(X)$. We can use the natural maps (2.1) and (2.2) to construct a natural map $I(X) \rightarrow \Omega(I(S(X)))$ of towers, and this leads in the usual way to a stable version $\left\{E_{r}(X ; E)\right\}$ of the above spectral sequence. (See Mosher and Tangora [16, Chapter 18], for an exposition of the case $E=K\left(Z_{p}\right)$.) Convergence of $\left\{E_{r}(X ; E)\right\}$ is the same as convergence of $\left\{U E_{r}(X ; E)\right\}$ with $\pi_{*}(X)$ replaced by $\pi_{*}^{s}(X)$, the stable homotopy of $X$.

This stable spectral sequence generalizes. For any pointed spaces $Y$ and $Z$, let $\{Y, Z\}^{q}=\lim _{i \rightarrow \infty}\left[Y, \Omega^{i-q}\left(S^{i}(Z)\right)\right]$ where $[$,$] denotes (pointed)$ homotopy classes of maps. Applying the functor $\{Y,\}^{*}$, where $Y$ is a finite dimensional CW complex, to the towers $I\left(S^{k}(X)\right)$, we obtain a stable spectral sequence $\left\{E_{r}(Y, X ; E)\right\}$ where 


$$
E_{1}^{s, t}(Y, X ; E)=\left[Y, \Omega^{t-s+k}\left(E\left(S^{k}(X)_{s}\right)\right)\right]
$$

for large $k$.

Convergence is as follows:

If $\pi_{0}(E) \approx Z,\left\{E_{r}(Y, X ; E)\right\} \Rightarrow\{Y, X\}^{*}$.

If $\pi_{0}(E) \approx Z_{p}$ and $Y$ is finite, $\left\{E_{r}(Y, X ; E)\right\} \Rightarrow_{C_{p}}\{Y, X\}^{*}$.

REMARK. If $E$ is a ring spectrum and $X$ is $E$-complete in the sense of [1, $\S 14]$ (our restriction that $\pi_{*}(X)$ be of finite type is getting waived here), then $\left\{E_{r}(Y, X ; E)\right\}$ is the spectral sequence defined by Adams in $[1, \S 15]$. It should be pointed out that Adams defined his spectral sequence for more general spectra than we are working with in this paper.

3. The resolution $I(X)$ through dimension $t$. Throughout this section, we assume $X$ is $n$-connected where $n \geqslant 1$. By a resolution with respect to $E$ through dimension $t$, we mean a diagram

$I^{t}(X)$

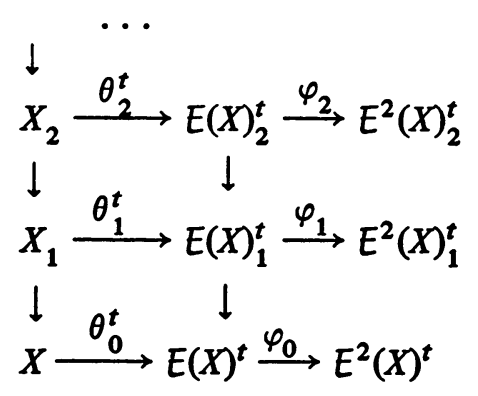

constructed as follows.

The spaces $X_{i}$ and maps $X_{i+1} \rightarrow X_{i}$ are from $I(X)$. We define the remaining spaces and maps of $I^{t}(X)$ by induction on the subscript $i$. For a space $A$, let $(A)^{t}$ denote a space such that $\pi_{i}\left((A)^{t}\right)=0$ for $i>t$ and there is a $(t+1)$ equivalence $A \rightarrow(A)^{t}$. Set $E(X)^{t}$ equal to $(E(X))^{t}$, and $E^{2}(X)^{t}$ equal to $\left(E\left(E(X)^{t}\right)\right)^{t+1}$. Let $\varphi_{0}: E(X)^{t} \rightarrow E^{2}(X)^{t}$ be the composite of the natural inclusion $E(X)^{t} \rightarrow E\left(E(X)^{t}\right)$ and the natural map $E\left(E(X)^{t}\right) \rightarrow E^{2}(X)^{t}$.

Assuming everything defined for $i \leqslant k$, take $E(X)_{k+1}^{t} \rightarrow E(X)_{k}^{t}$ to be the principal fibration induced by $\varphi_{k}$, and define $\theta_{k+1}^{t}$ to be the map induced by the commutative diagram

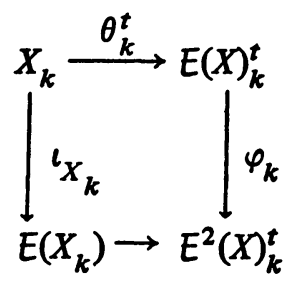


where the bottom map is the composite of $E\left(\theta_{k}^{t}\right)$ and the natural map $E\left(E(X)_{k}^{t}\right) \rightarrow E^{2}(X)_{k}^{t}$. Finally, set $E^{2}(X)_{k+1}^{t}$ equal to $\left(E\left(E(X)_{k+1}^{t}\right)\right)^{t+1}$ and let $\varphi_{k+1}$ be the composite of the natural inclusion $E(X)_{k+1}^{t} \rightarrow E\left(E(X)_{k+1}^{t}\right)$ and the map $E\left(E(X)_{k+1}^{t}\right) \rightarrow E^{2}(X)_{k+1}^{t}$.

Theorem 3.1. $\pi_{j}\left(E(X)_{i}^{\dagger}\right)=0$ for $j>t$ and all $i$. If $t<2 n$, there is a $(t+1)$-equivalence $\beta_{i}: E\left(X_{i}\right) \rightarrow E(X)_{i}^{t}$ for each $i$, such that $\beta_{i}{ }^{\circ}{ }^{\prime} X_{i}$ is homotopic to $\theta_{i}^{t}$.

RFMARKs. Observe that the space $E(X)_{i}^{t}$ depends only on $E(X)^{t}$ and if $t<2 n, E(X)_{i}^{t}$ has the same homotopy type as $\Omega^{k}\left(E\left(S^{k}(X)\right)_{i}^{t+k}\right)$ for any $k$. Hence if $\operatorname{dim} Y+r-s \leqslant t<2 n, E_{1}^{s, r}(Y, X ; E)$ can be identified with $\left[Y, \Omega^{r-s}\left(E(X)_{s}^{t}\right)\right]$.

The construction of $I^{t}(X)$ is motivated by the Bousfield-Kan approach to resolutions [5].

The proof of 3.1 is by induction on $i$. The case $i=0$ is trivial. Assume then that everything has been established for $i \leqslant k$. The assertion that $\pi_{j}\left(E(X)_{k+1}^{t}\right)=0$ for $j>t$ is clear. Consider the following commutative diagram:

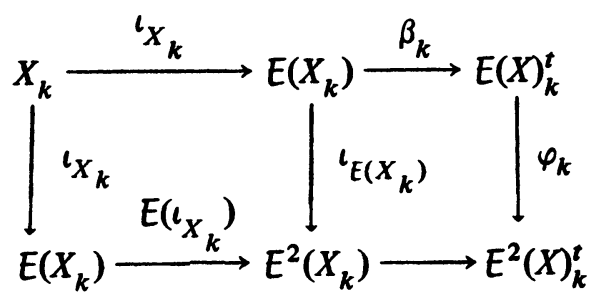

The left square in (3.2) induces a map $\theta_{k+1}: X_{k+1} \rightarrow E\left(X_{k}\right)_{1}$ and the right square a map $\delta_{k+1}: E\left(X_{k}\right)_{1} \rightarrow E(X)_{k+1}^{t}$ such that $\delta_{k+1} \circ \theta_{k+1}$ is homotopic to $\theta_{k+1}^{t}$.

LEMMA 3.3. If $t<2 n$, then $\delta_{k+1}$ is $a(t+1)$ equivalence.

LEMMA 3.4. If $t<2 n$, there is a homotopy equivalence $\mu$ : $\left(E\left(X_{k+1}\right)\right)^{t}$ $\rightarrow\left(E\left(X_{k}\right)_{1}\right)^{t}$ such that

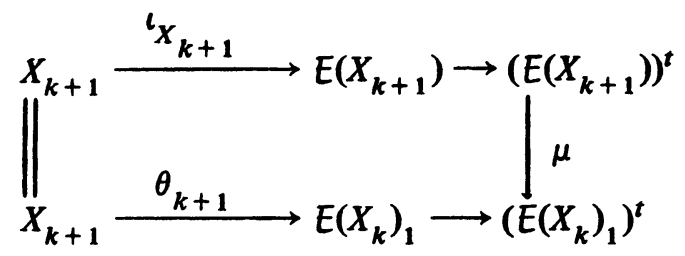

is homotopy commutative. 
Before proving 3.3 and 3.4, we complete the proof of 3.1. By 3.3, we may identify $E(X)_{k+1}^{t}$ with $\left(E\left(X_{k}\right)_{1}\right)^{t}$. Then by 3.4 , there is a $(t+1)$-equivalence $\beta_{k+1}: E\left(X_{k+1}\right) \rightarrow E(X)_{k+1}^{t}$ such that $\beta_{k+1}{ }^{\circ} \iota_{X_{k+1}}$ is homotopic to $\delta_{k+1} \circ$ $\theta_{k+1}$.

PROOF OF 3.3. We assume $k=0$ for simplicity of notation; the proof for arbitrary $k$ is the same. $\delta_{1}, \beta_{0}$ and the natural map $E^{2}(X) \rightarrow E^{2}(X)^{t}$ define a homomorphism from the homotopy exact sequence of the fibration $E(X)_{1} \rightarrow$ $E(X) \rightarrow E^{2}(X)$ into the homotopy exact sequence of the fibration $E(X)_{1}^{t} \rightarrow$ $E(X)^{t} \rightarrow E^{2}(X)^{t}$. Applying a 5-lemma type argument, we see that $\delta_{1}$ induces isomorphisms of homotopy groups in dimensions $<t$, and that $\left(\delta_{1}\right)_{*}: \pi_{t}\left(E(X)_{1}\right)$ $\rightarrow \pi_{t}\left(E(X)_{1}^{t}\right)$ is an isomorphism if

$$
\pi_{t+1}(E(X)) \rightarrow \pi_{t+1}\left(E^{2}(X)\right) \rightarrow \pi_{t+1}\left(E^{2}(X)^{t}\right)
$$

is exact where the first homomorphism is induced by the natural inclusion and the second by the natural map $E^{2}(X) \rightarrow E^{2}(X)^{t}$. Since $t<2 n$, we may make the following identifications:

$$
\begin{aligned}
& \pi_{t+1}(E(X)) \text { with } \pi_{t+k+l+1}\left(X \wedge E_{k} \wedge S^{l}\right), \\
& \pi_{t+1}\left(E^{2}(X)\right) \text { with } \pi_{t+k+l+1}\left(X \wedge E_{k} \wedge E_{l}\right), \\
& \pi_{t+1}\left(E^{2}(X)^{t}\right) \text { with } \pi_{t+k+l+1}\left(\left(X \wedge E_{k}\right)^{t+k} \wedge E_{l}\right),
\end{aligned}
$$

for large $k, l$. Then (3.5) becomes

$$
\begin{aligned}
\pi_{t+k+l+1}\left(X \wedge E_{k} \wedge S^{l}\right) & \stackrel{a_{*}}{\longrightarrow} \pi_{t+k+l+1}\left(X \wedge E_{k} \wedge E_{l}\right) \\
& \stackrel{b_{*}}{\longrightarrow} \pi_{t+k+l+1}\left(\left(X \wedge E_{k}\right)^{t+k} \wedge E_{l}\right)
\end{aligned}
$$

for large $k$ and $l$, where $a$ is obtained by smashing $\iota_{l}: S^{l} \rightarrow E_{l}$ with $X \wedge E_{k}$ and $b$ is obtained by smashing $\left(X \wedge E_{k}\right) \rightarrow\left(X \wedge E_{k}\right)^{t+k}$ with $E_{l}$.

We may assume that the l-skeleton of $E_{l}$ is $S^{l}$ and that $\left(X \wedge E_{k}\right)^{t+k}$ is obtained from $X \wedge E_{k}$ by attaching cells of dimension $\geqslant t+k+2$. Then $\left(X \wedge E_{k}\right)^{t+k} \wedge E_{l}$ is obtained from $X \wedge E_{k} \wedge E_{l}$ by attaching cells of dimension $\geqslant t+k+l+2$ and it is clear that the attaching process kills precisely the image of $a_{*}$ in dimension $t+k+l+1$.

Proof OF 3.4. Again we assume $k=0$ for simplicity of notation. We need the following lemma for which the reader can easily supply a proof.

LEMMA 3.7. Let $P_{g} \rightarrow A$ be the principal fibration induced by the map $g: A \rightarrow B$. Then there is a natural map $\alpha: E\left(P_{g}\right) \rightarrow P_{E(g)}$ which induces isomorphism of homotopy groups through dimension $2 n-1$ if $B$ and $P_{g}$ are each n-connected. Moreover, $\alpha{ }^{\circ} \iota_{P_{g}}$ is the map induced by the following commutative square: 


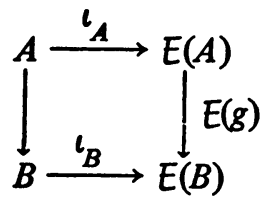

Taking $g=\iota_{X}$ in Lemma 3.7, let $\psi_{1}=\alpha{ }^{\circ} \iota_{X_{1}}: X_{1} \rightarrow P_{E\left(\iota_{X}\right)}$. We wish to compare $\psi_{1}$ and $\theta_{1}$. To that end, we introduce the following commutative diagram:

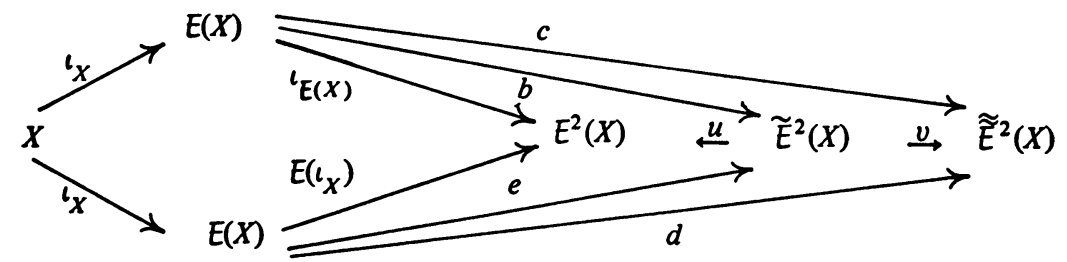

$$
\widetilde{E}^{2}(X)=\lim _{m \rightarrow \infty} \Omega^{m}\left(\left(\Omega^{m}\left(X \wedge E_{m}\right)\right) \wedge E_{m}\right)
$$

under the composite of natural inclusions

$$
\begin{aligned}
\Omega^{m}\left(\left(\Omega^{m}\left(X \wedge E_{m}\right)\right) \wedge E_{m}\right) & \rightarrow \Omega^{m}\left(\left(\Omega^{m+1}\left(X \wedge E_{m+1}\right)\right) \wedge E_{m}\right) \\
& \rightarrow \Omega^{m+1}\left(\left(\Omega^{m+1}\left(X \wedge E_{m+1}\right)\right) \wedge E_{m+1}\right) .
\end{aligned}
$$

$\widetilde{E}^{2}(X)=\lim _{m \rightarrow \infty} \Omega^{2 m}\left(X \wedge E_{m} \wedge E_{m}\right)$ under the embedding

$$
\Omega^{2 m}\left(X \wedge E_{m} \wedge E_{m}\right) \rightarrow \Omega^{2 m+2}\left(X \wedge E_{m+1} \wedge E_{m+1}\right)
$$

which takes a $2 m$-loop $\sigma: S^{2 m} \rightarrow X \wedge E_{m} \wedge E_{m}$ to the composite $(2 m+2)$ loop

$$
\begin{aligned}
S^{2 m+2} & \stackrel{\sigma \wedge 1}{\longrightarrow} X \wedge E_{m} \wedge E_{m} \wedge S^{1} \wedge S^{1} \\
& \rightarrow X \wedge E_{m} \wedge S^{1} \wedge E_{m} \wedge S^{1} \stackrel{1 \wedge \epsilon \wedge \epsilon}{\longrightarrow} X \wedge E_{m+1} \wedge E_{m+1}
\end{aligned}
$$

where the middle map takes $\left[x, e, e^{\prime}, t_{1}, t_{2}\right]$ to $\left[x, e, t_{2}, e^{\prime}, t_{1}\right]$.

$u$ and $v$ are the obvious maps. $b$ is the direct limit of the natural inclusions $\Omega^{m}\left(X \wedge E_{m}\right) \rightarrow \Omega^{m}\left(\left(\Omega^{m}\left(X \wedge E_{m}\right)\right) \wedge E_{m}\right) . e$ is obtained by applying the functor $\Omega^{m}\left(\bigwedge E_{m}\right)$ to the natural inclusion $X \rightarrow \Omega^{m}\left(X \wedge E_{m}\right)$ and passing to the limit. $c=v \circ b$ and $d=v \circ e$.

The commutative square containing $\iota_{X}, c, d \iota_{X}$ induces maps $\widetilde{\theta}_{1}: X_{1}$ $\rightarrow P_{c}, \widetilde{\Psi}_{1}: X_{1} \rightarrow P_{d}$. Since $u$ is a weak homotopy equivalence and $v$ induces 
isomorphisms of homotopy groups in dimensions $\leqslant 2 n$, if $t<2 n, u$ and $v$ induce homotopy equivalences $\mu_{1}:\left(P_{E\left(\iota_{X}\right)}\right)^{t} \rightarrow\left(P_{d}\right)^{t}$ and $\mu_{2}:\left(E(X)_{1}\right)^{t} \rightarrow$ $\left(P_{c}\right)^{t}$ such that

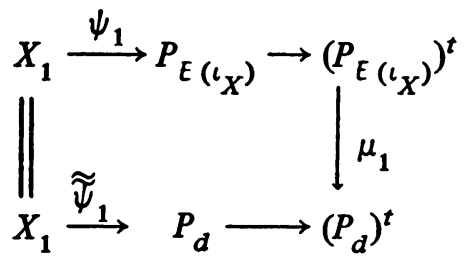

(3.9)

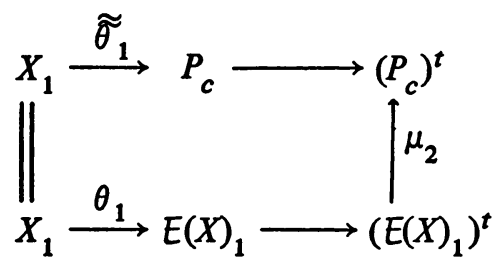

are homotopy commutative.

Let $t_{2}: \widetilde{E}^{2}(X) \rightarrow \widetilde{E}^{2}(X)$ map $\sigma: S^{m} \wedge S^{m} \rightarrow X \wedge E_{m} \wedge E_{m}$ to the composite

$$
S^{m} \wedge S^{m} \stackrel{\alpha}{\longrightarrow} S^{m} \wedge S^{m} \stackrel{\sigma}{\longrightarrow} X \wedge E_{m} \wedge E_{m} \stackrel{\gamma}{\longrightarrow} X \wedge E_{m} \wedge E_{m}
$$

where $\alpha$ reverses the order of the factors $S^{m}$ and $\gamma$ reverses the order of the factors $E_{m} \cdot t_{2}$ is a homeomorphism and $t_{2} \circ c=d, t_{2} \circ d=c$. Hence $t_{2}$ defines a homeomorphism $\bar{\mu}: P_{d} \rightarrow P_{c}$ such that $\bar{\mu} \circ \widetilde{\Psi}_{1}=\widetilde{\widetilde{\theta}}_{1}$. Combining this fact with (3.9) and Lemma 2.7 (in the case $g=\iota_{X}$ ) completes the proof of 3.4.

4. Resolutions in $T B$. In this section we define an analogue of the spectral sequence $\left\{E_{r}(Y, X ; E)\right\}$ for enumerating liftings by carrying out the analogues of the constructions of $\S 2$ in the category $T B$ of $B$-sectioned spaces. For a thorough exposition of this category, see McClendon [13]. We outline briefly the properties of $T B$ which we need.

For a similar approach using the semisimplicial category, see Anderson [2].

A $B$-sectioned space is a space $W$ together with a continuous projection $\hat{w}: W \rightarrow B$ and a canonical cross-section $\check{w}: B \rightarrow W$ of $\hat{w}$. We normally denote this $B$-sectioned space simply by $W$ and use the canonical cross-section $\check{w}$ to identify $B$ with a subspace of $W$. The cross-section $\breve{w}$ is to be thought of as a continuous choice of basepoint in each inverse image $\hat{w}^{-1}(b)$ for $b \in$ B. A map in $T B$ from $W \in T B$ to $W^{\prime} \in T B$ is a continuous map $W \rightarrow W^{\prime}$ covering the identity map of $B$ and mapping the canonical cross-section of $W$ to that of $W^{\prime}$. 
If $f: Y \rightarrow B$ is a map, we denote by $[Y, W]_{f}$ the set of (vertical) homotopy classes of liftings of $f$ to $W .[Y, W]_{f}$ has a natural zero element, the homotopy class of $\breve{w} f$.

In general, if $W$ is a $B$-sectioned space, it is not assumed that the projection $\hat{w}: W \rightarrow B$ is a fibration (of any kind). Since for many arguments some such assumption is necessary, we introduce the subcategory $T B$ (fib) of $T B$. A space $W \in T B$ is in $T B$ (fib) if the projection $(W, B) \rightarrow(B, B)$ is a weak pair fibration. (See the appendix for the definition of weak pair fibration.) $A$ map $\alpha$ in $T B$ from $W \in T B$ (fib) to $W^{\prime} \in T B$ (fib) is in $T B$ (fib) if the map $(W, B) \rightarrow\left(W^{\prime}, B\right)$ of pairs defined by $\alpha$ is a weak pair fibration.

If $W \in T B$, there is a canonical $B$-principal fibration (path-loop) fibration

$$
\Omega_{B}(W) \rightarrow P_{B}(W) \stackrel{\pi}{\rightarrow} W
$$

in $T B$, where $P_{B}(W)$ (resp., $\Omega_{B}(W)$ ) is the union of the path spaces (resp., loop spaces) of the inverse images $\hat{w}^{-1}(b)$ for $b \in B$, topologized as a subspace of $W^{I}$, and $\pi$ is evaluation at $1 \in I$. If $W \in T B(\mathrm{fib})$, then $P_{B}(W)$, $\Omega_{B}(W)$, and $\pi: P_{B}(W) \rightarrow W$ are also in $T B(\mathrm{fib})$.

If $h: Z \rightarrow W$ is a map, where $Z$ is not assumed to be a $B$-sectioned space but $W$ is, the $B$-principal fibration $\bar{P}_{h} \rightarrow Z$ induced by $h$ is the pullback of $P_{B}(W) \rightarrow W$ by $h$. Induced $B$-principal fibrations have many of the properties in $T B$ that ordinary induced principal fibrations have in the category of pointed spaces. For example, if $g: Y \rightarrow Z$ is a lifting of $f: Y \rightarrow B$, then $g$ lifts to $\bar{P}_{h}$ if and only if $[h g]=0$ in $[Y, W]_{f}$.

Now assume that $Z \in T B, h \in T B$, and $f: Y \rightarrow B$ is any map. Then $\bar{P}_{h} \in T B$ and there is an exact sequence of pointed sets

$$
\left[Y, \Omega_{B}(W)\right]_{f} \rightarrow\left[Y, \bar{P}_{h} I_{f} \rightarrow[Y, Z]_{f} \rightarrow[Y, W]_{f}\right.
$$

If $Z=\Omega_{B}\left(Z^{\prime}\right), W=\Omega_{B}\left(W^{\prime}\right)$, and $h=\Omega_{B}\left(h^{\prime}\right)$ where $h^{\prime}: Z^{\prime} \rightarrow W^{\prime}$ is a map in $T B,(4.1)$ becomes an exact sequence of groups and homomorphisms.

Notice that $P_{B}$ and $\Omega_{B}$ are just "fiberwise" path and loop functors. Many other constructions on pointed spaces can be carried out "fiberwise" to give analogous constructions in $T B$. For example, if $W \in T B$, we can form the "fiberwise" (reduced) suspension $S_{B}(W)$ of $W$ (topologized as a quotient space of $W \times I)$, and there is an obvious map $W \rightarrow \Omega_{B}\left(S_{B}(W)\right)$. For any map $f: Y \rightarrow B$, we set

$$
\{Y, W\}_{f}^{q}=\lim _{i \rightarrow \infty}\left[Y, \Omega_{B}^{i-q}\left(S_{B}^{i}(W)\right)\right]_{f}
$$


If $W \in T B$ (fib) has $n$-connected fiber and $Y$ is a $C W$-complex of dimension $<2 n$, then $[Y, W]_{f}=\{Y, W\}_{f}^{0}$.

Throughout the rest of this section, we assume that $W \in T B$ (fib), that $(W, B)$ has the homotopy type of a $\mathrm{CW}$ pair, and that the homotopy groups of the fibers of the projection $W \rightarrow B$ are finitely generated. For each spectrum $E=\left\{E_{m}\right\}$ satisfying condition (a)-(e) in $\S 2$ and each finite dimensional $\mathrm{CW}$ complex $Y$, we now construct a spectral sequence of Adams type converging to a quotient group of $\{Y, W\}_{f}^{*}$.

Let $E_{B}(W)=\lim _{m \rightarrow \infty} \Omega_{B}^{m}\left(W \wedge_{B} E_{m}\right) . W \wedge_{B} E_{m}$ is the $B$-sectioned space whose fibers are the fibers of $W$ smashed with $E_{m}$. (W $W \wedge_{B} E_{m}$ is topologized as a quotient space of $W \times E_{m}$.) There is a natural inclusion $\imath_{W}: W \rightarrow E_{B}(W)$. By Theorem 8.7 of the appendix, if $B$ is locally compact, $E_{B}(W) \in T B$ (fib).

The resolution $I_{B}(W)$ of $W$ with respect to $E$ is the following tower of $B$-principal fibrations over $W$.

$I_{B}(W)$

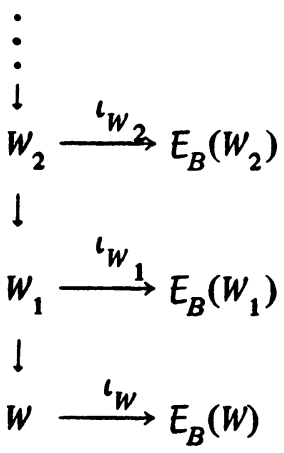

If $B$ is locally compact, it follows from previous remarks and the appendix that all of the spaces and all of the vertical maps in $I_{B}(W)$ are in $T B$ (fib). For that reason, we assume from now on that $B$ is locally compact. All of the subsequent results can be obtained without this assumption with a little more effort.

There is a map of towers $I_{B}(W) \rightarrow \Omega_{B}\left(I_{B}\left(S_{B}(W)\right)\right)$ analogous to the map $I(X) \rightarrow \Omega(I(S(X)))$ described in $\S 2$. Applying the functor $\{Y\}_{f}^{*}$ to the resolutions $I_{B}\left(S^{k}(W)\right)$ and piecing together exact sequences like (4.1) to give an exact couple, we obtain a stable spectral sequence $\left\{E_{r}(Y, W ; E)_{f}\right\}$ where

$$
E_{1}^{s, t}(Y, W ; E)_{f}=\left[Y, \Omega_{B}^{t-s+k}\left(E_{B}\left(S_{B}^{k}(W)_{s}\right)\right)\right]_{f}
$$

for large $k$.

Convergence is as follows:

If $\pi_{0}(E) \approx \mathbf{Z},\left\{E_{r}(Y, W ; E)_{f}\right\} \Rightarrow\{Y, W\}_{f}^{*}$. 
If $\pi_{0}(E) \approx Z_{p}$ and $Y$ is finite, $\left\{E_{r}(Y, W ; E)_{f}\right\} \Rightarrow_{c_{p}}\{Y, W\}_{f}^{*}$.

Convergence is again first established for $E=K(\mathrm{Z})$ or $K\left(\mathrm{Z}_{p}\right)$ and then convergence for general $E$ follows by comparison using the map $E \rightarrow K\left(\pi_{0}(E)\right)$. Convergence for $E=K(Z)$ is a consequence of Lemma 2.4. Convergence for $E=K\left(Z_{p}\right)$ can be proved by mimicking, in the category $T B$, the proof of convergence of $\left\{E_{r}\left(Y, X ; K\left(Z_{p}\right)\right)\right\}$ as given in Chapter 18 of [16].

4.3. REMARK. Let $T \rightarrow B$ be a fibration with $(n-1)$-connected fiber. Let $S_{B}^{\prime}(T)$ denote the fiberwise unreduced suspension of $T \rightarrow B$; by means of the south pole section of $S_{B}^{\prime}(T) \rightarrow B$, we regard $S_{B}^{\prime}(T)$ as a $B$-sectioned space. If $Y$ has dimension $\leqslant 2 n-2$ and $f: Y \rightarrow B$ lifts to $T$, then $\left\{Y, S_{B}^{\prime}(T)\right\}_{f}^{-1}$ is in one-to-one correspondence with $[Y, T]_{f}$. (See Larmore $[9, \S 4]$ or Becker $[3, \S 7]$.)

When $T \rightarrow B$ is orientable (see $\S 7$ of this paper) with respect to $K\left(Z_{p}\right)$ for some prime $p$, the spectral sequence $\left\{E_{r}\left(Y, S_{B}^{\prime}(T) ; K\left(Z_{p}\right)\right)_{f}\right\}$ is essentially the spectral sequence constructed by McClendon in [12] and Meyer in [15].

5. The resolution $I_{B}(W)$ through dimension $t$. We continue to assume that $W \in T B$ (fib) and that $(W, B)$ has the homotopy type of a $C W$ pair. Assume additionally that each fiber $F_{b}$ of the projection $W \rightarrow B$ is $n$-connected where $n \geqslant 1$.

$(W)_{B}^{t}$ will denote a space in $T B$ (fib) having fiber $\left(F_{b}\right)^{t}$ over $b \in B$ such that $\left((W)_{B}^{t}, B\right)$ has the homotopy type of a $C W$ pair and there is a map $W \rightarrow(W)_{B}^{t}$ in $T B$ which is a $(t+1)$-equivalence on fibers. $(W)_{B}^{t}$ can be constructed for any $t$ using Postnikov resolutions in the category TB. (See [14]. Strictly speaking the Postnikov resolutions that we are talking about are not constructed in [14]; however, the techniques needed for constructing them can be found there.)

By a resolution in $T B$ with respect to $E$ through dimension $t$, we mean a diagram:

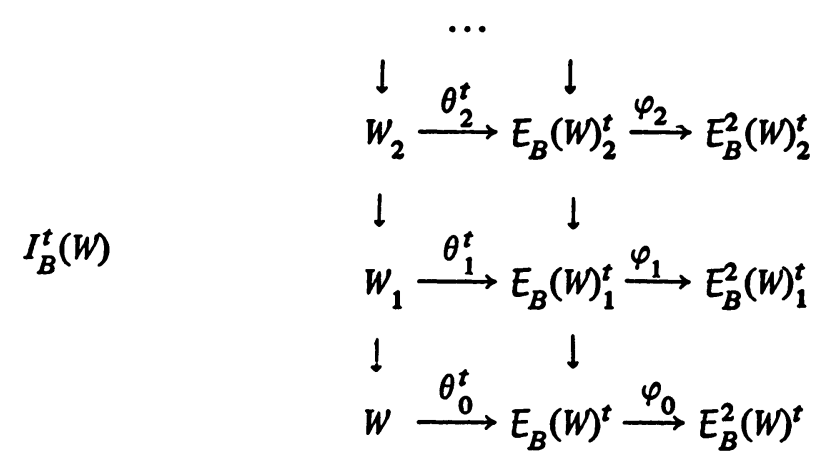


$I_{B}^{t}(W)$ is built up exactly as $I^{t}(X)$ is built up; simply replace $E()$ by $E_{B}($ ), ()$^{t}$ by ()$_{B}^{t}$ and work in $T B$. All of the spaces and all of the vertical maps in $I_{B}^{t}(W)$ are in $T B(\mathrm{fib})$.

Theorem 5.1. Assume $t<2 n$. Then for each $i$ there is a map $\beta_{i}$ : $E_{B}\left(W_{i}\right) \rightarrow E_{B}(W)_{i}^{t}$ in $T B$ which is $a(t+1)$ equivalence on fibers, such that $\beta_{i} \circ \iota_{W_{i}}$ is homotopic to $\theta_{i}^{t}$ in $T B$.

The proof is the same as the proof of Theorem 3.1 except that we work in $T B$. It must be checked that the analogues $\widetilde{E}_{B}^{2}(W)$ and $\widetilde{E}_{B}^{2}(W)$ in $T B$ of $\widetilde{E}^{2}(X)$ and $\widetilde{E}^{2}(X)$ are actually in $T B$ (fib), but this is easily done using the appendix.

REMARK. Observe that if $t<2 n, E_{B}(W)_{i}^{t}$ is fiber homotopically equivalent as a $B$-sectioned space to $\Omega_{B}^{k}\left(E_{B}\left(S_{B}^{k}(W)\right)_{i}^{t+k}\right)$ for any $k$. Hence if $\operatorname{dim} Y+r-$ $s \leqslant t<2 n, E_{1}^{s, r}(Y, W ; E)_{f}$ can be identified with $\left[Y, \Omega_{B}^{r-s}\left(E_{B}(W)_{s}^{t}\right)\right]_{f^{*}}$

6. The existence problem for liftings. Results up until now have been concerned with enumerating liftings. We turn now to the problem of determining whether a given map $f: Y \rightarrow B$ lifts to $T$ where $T \rightarrow B$ is a fibration with $(n-1)$-connected fiber.

Recall that $S_{B}^{\prime}(T)$ is the fiberwise unreduced suspension of $T \rightarrow B$ and that we regard $S_{B}^{\prime}(T)$ as a $B$-sectioned space by taking the south pole section to be the canonical cross-section. By Corollary 8.6 of the appendix, $S_{B}^{\prime}(T) \in$ $T B$ (fib). We will formulate an obstruction theory based on the diagram $I_{B}^{t}\left(S_{B}^{\prime}(T)\right)$.

Let $s_{1}: B \rightarrow S_{B}^{\prime}(T)$ be the north pole section. Restricting the tower $I_{B}\left(S_{B}^{\prime}(T)\right)$ to $S_{1}(B)$ yields the following tower of $B$-principal fibrations where the map $T_{i} \rightarrow E_{B}\left(S_{B}^{\prime}(T)_{i}\right)$ is the restriction of the map $S_{B}^{\prime}(T)_{i} \rightarrow E_{B}\left(S_{B}^{\prime}(T)_{i}\right)$ from $I_{B}\left(S_{B}^{\prime}(T)\right)$.

$I I(T)$

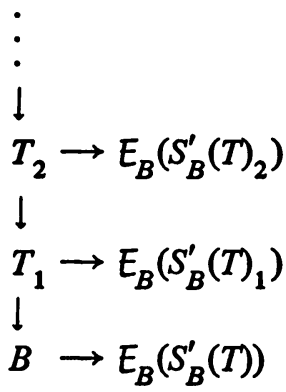

Similarly restricting $I_{B}^{t}\left(S_{B}^{\prime}(T)\right)$, we obtain 
$I^{t}(T)$

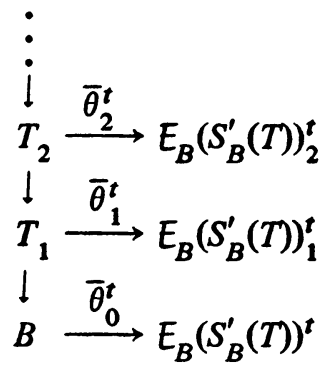

where $\bar{\theta}_{i}^{t}$ is the restriction of $\theta_{i}^{t}$.

Assume $Y$ is a $\mathrm{CW}$ complex of dimension $\leqslant 2 n-1$. The single obstruction $O(f)$ to lifting $f$ to $T$ is the homotopy class of $s_{1} \circ f$ in $\left\{Y, S_{B}^{\prime}(T)\right\}_{f}^{0}$. $O(f)$ was introduced by Larmore [9] and Becker [4]. (Becker defined it only for sphere bundles and called it an Euler class.) It is called the single obstruction because $f$ lifts to $T$ if and only if $O(f)=0$.

We use $O(f)$ to prove:

Theorem 6.1. Assume $\operatorname{dim} Y \leqslant 2 n-1$. If $\pi_{0}(E) \approx Z, f$ lifts to $T$ if and only if $f$ lifts to $T_{m}$ where $m=\operatorname{dim} Y-n$. If $\pi_{0}(E) \approx Z_{p}$ and $Y$ is finite, $O(f)$ is zero or has finite order prime to $p$ if and only if $f$ lifts to each $T_{i}$.

Proof. By the relationship between $I I(T)$ and $I_{B}\left(S_{B}^{\prime}(T)\right), f$ lifts to $T_{i}$ if and only if $O(f)$ is in the image of $\left[Y, S_{B}^{\prime}(T)_{i}\right]_{f} \rightarrow\left[Y, S_{B}^{\prime}(T)\right]_{f}$. Hence Theorem 6.2 follows from the convergence of the spectral sequence $\left\{E_{r}\left(Y, S_{B}^{\prime}(T) ; E\right)_{f}\right\}$. (If $\pi_{0}(E) \approx Z$, we must invoke Lemma 2.4 and make use of the map of towers induced by $E \rightarrow K\left(\pi_{0}(E)\right)$.)

If $\operatorname{dim} Y \leqslant t<2 n$, we define the $i$ th obstruction to lifting $f$ to be

$$
O^{i}(f)=\left\{\left[\bar{\theta}_{i}^{t} \circ g\right] \in\left[Y, E_{B}\left(S_{B}^{\prime}(T)\right)_{i}^{t}\right]_{f} \mid g: Y \rightarrow T_{i} \text { is a lifting of } f\right\} .
$$

By the remark following Theorem 5.1, we may identify $\left[Y, E_{B}\left(S_{B}^{\prime}(T)\right)_{i}^{t}\right]_{f}$ with $E_{1}^{i, i}\left(Y, S_{B}^{\prime}(T) ; E\right)_{f}$. Then $O^{i}(f)$ is a coset of

$$
B_{\infty}^{i, i}=\bigcup_{r} B_{r}^{i, i}\left(Y, S_{B}^{\prime}(T) ; E\right)_{f},
$$

where $B$ denotes boundaries.

7. Orientability. We say that $W \in T B$ and $W^{\prime} \in T B$ are fiber homotopically equivalent as $B$-sectioned spaces if there are maps $\alpha: W \rightarrow W^{\prime}$ and $\beta$ : $W^{\prime} \rightarrow W$ in $T B$ such that $\alpha \beta$ and $\beta \alpha$ are homotopic to $1_{W^{\prime}}$ and $1_{W}$ respectively, through maps in $T B . W$ is fiber homotopically trivial as a $B$-sectioned space if $W$ is fiber homotopically equivalent as a $B$-sectioned space to $B \times F$ 
where $F$ is some pointed space. (It is assumed that the canonical cross-section of $B \times F$ has image $B \times *$.)

7.1. The fibration $T \rightarrow B$ is said to be orientable with respect to $E$ through dimension $t$ if $E_{B}\left(S_{B}^{\prime}(T)\right)^{t}$ is fiber homotopically trivial as a $B$-sectioned space.

Throughout the remainder of this section, we assume that $B$ is connected, $B$ has a basepoint, and $F$ is the fiber of $T \rightarrow B$ over the basepoint.

THEOREM 7.2. If $T \rightarrow B$ is orientable with respect to $E$ through dimension $t$, then in $I_{B}^{t}\left(S_{B}^{\prime}(T)\right)$, we may take $E_{B}\left(S_{B}^{\prime}(T)\right)_{i}^{t}$ to be $B \times E\left(S^{\prime}(F)\right)_{i}^{t}$ for each $i$.

REMARK. If $\operatorname{dim} Y+r-s \leqslant t<2 n$, Theorem 7.2 and the remark following Theorem 5.1 imply that

$$
E_{1}^{s, r}\left(Y, S_{B}^{\prime}(T) ; E\right)_{f}=\left[Y, \Omega^{r-s}\left(E\left(S^{\prime}(F)\right)_{s}^{t}\right)\right]
$$

if $T \rightarrow B$ is orientable with respect to $E$ through dimension $t$. In particular, $O^{i}(f)$ is a coset in $\left[Y, E\left(S^{\prime}(F)\right)_{i}^{t}\right]$. Theorem 3.4 of Gitler-Mahowald [7] is the special case in which $E=K\left(\mathbf{Z}_{2}\right)$.

Theorem 7.2 and the following remark should make calculations involving the spectral sequence $\left\{E_{r}\left(Y, S_{B}^{\prime}(T) ; E\right)_{f}\right\}$ and the obstructions $O^{i}(f)$ feasible in the orientable case. For example, McClendon [12] and Meyer [15] have shown that if, for some prime $p, T \rightarrow B$ is orientable with respect to $K\left(Z_{p}\right)$ through dimension $t$ for all $t$, then $E_{2}^{s, r}\left(Y, S_{B}^{\prime}(T) ; K\left(Z_{p}\right)\right)_{f}$ is

$$
\operatorname{Ext}_{A(B)}^{s, r}\left(H^{*}\left(S_{B}^{\prime}(T), B ; Z_{p}\right), H^{*}\left(Y ; Z_{p}\right)\right)
$$

where $A(B)=H^{*}(B) \circ A$ is the mod $p$ Massey-Peterson algebra [11].

Proof of 7.2. We establish the theorem by induction on $i$. By assumption, we may take $E_{B}\left(S_{B}^{\prime}(T)^{t}\right)=B \times E\left(S^{\prime}(F)\right)^{t}$, starting the induction. If $E_{B}\left(S_{B}^{\prime}(T)\right)_{i}^{t}=B \times E\left(S^{\prime}(F)\right)_{i}^{t}$, it is clear from the construction of $I_{B}^{t}\left(S_{B}^{\prime}(T)\right)$ that we may take $E_{B}^{2}\left(S_{B}^{\prime}(T)\right)_{i}^{t}=B \times E^{2}\left(S^{\prime}(F)\right)_{i}^{t}$ and the map $E_{B}\left(S_{B}^{\prime}(T)\right)_{i}^{t} \rightarrow$ $E_{B}^{2}\left(S_{B}^{\prime}(T)\right)_{i}^{t}$ to be the product of the identity on $B$ and the map $E\left(S^{\prime}(F)\right)_{i}^{t} \rightarrow$ $E^{2}\left(S^{\prime}(F)\right)_{i}^{t}$ from $I^{t}\left(S^{\prime}(F)\right)$. This implies that $E_{B}\left(S^{\prime}(T)\right)_{i+1}^{t}=B \times E\left(S^{\prime}(F)\right)_{i+1}^{t}$.

The following theorem gives a useful criterion for determining when a fibration is orientable and also describes the relationship between our definition and other definitions of orientability.

THEOREM 7.3. Let $R$ be a ring spectrum and $E$ an R-module spectrum. If there is a map $U:\left(S_{B}^{\prime}(T), B\right) \rightarrow\left(R\left(S^{\prime}(F)\right)^{t}, *\right)$ such that the restriction of $U$ to the fiber $\left(S^{\prime}(F), *\right)$ is homotopic to the natural map $\left(S^{\prime}(F), *\right) \rightarrow$ 
$\left(R\left(S^{\prime}(F)\right)^{t}, *\right)$, then $T \rightarrow B$ is orientable with respect to $E$ through dimension $t$.

(For the definitions of ring spectrum and R-module spectrum, see [4, pp. 571 and 576].)

Proof. $U$ defines a map $\left(E_{B}\left(S_{B}^{\prime}(T)\right)^{t}, B\right) \rightarrow\left(E\left(R\left(S^{\prime}(F)\right)^{t}\right)^{t}, *\right)$ and the pairing from $(R, E)$ to $E$ defines a map $\left(E\left(R\left(S^{\prime}(F)\right)^{t}\right)^{t}, *\right) \rightarrow\left(E\left(S^{\prime}(F)\right)^{t}, *\right)$. The composite of these two maps is a map $\left(E_{B}\left(S_{B}^{\prime}(T)\right)^{t}, B\right) \rightarrow\left(E\left(S^{\prime}(F)\right)^{t}, *\right)$ which, when restricted to a fiber, is a homotopy equivalence of pointed spaces. By a generalization (8.2 in the appendix) of a theorem of Dold [6], $E_{B}\left(S_{B}^{\prime}(T)\right)^{t}$ is fiber homotopically trivial as a $B$-sectioned space.

REMARK. It is easy to see that if $T \rightarrow B$ is orientable with respect to $R$ through dimension $t$, then there must exist such a map $U$.

EXAMPLE 1. Suppose $F$ has the homotopy type of $S^{n}$ and $E$ is a ring spectrum. Let $G=\left\{G_{m}\right\}$ be the ring spectrum with $G_{m}=\left(E_{m}\right)^{m+t}$. Then $T \rightarrow B$ is orientable with respect to $E$ through dimension $t$ if and only if there is an element $\bar{U} \in G^{n+1}\left(S_{B}^{\prime}(T), B\right)$ such that $\bar{U} \mid\left(S^{\prime}(F), *\right)$ generates $G^{*}\left(S^{\prime}(F), *\right)$ as a $G^{*}(*)$-module.

EXAMPLe 2. If $F$ is arbitrary and $E=K\left(\mathrm{Z}_{p}\right), p$ prime, then $T \rightarrow B$ is orientable with respect to $E$ through dimension $t$ if and only if every element in $H^{i}\left(F ; Z_{p}\right)$ for $i \leqslant t-1$ is transgressive.

We can easily describe an obstruction to orientability which generalizes the first SW class of a sphere bundle. For any pointed space $(X, *)$ having the homotopy type of a pointed CW complex, let $B(X)$ be the classifying space for fiber homotopy equivalence classes of fibrations with fiber of the homotopy type of $X$, and let $G(X) \rightarrow B(X)$ be the universal fibration.

It is not difficult to show that $G(X)$ is the classifying space for fiber homotopy equivalence classes of weak pair fibrations with fiber pair of the homotopy type of $(X, *)$. That is, there is $M \in T G(X)$ (fib) such that for every $N \in T Y$ (fib) where $Y$ has the homotopy type of a CW complex and the fiber pair of $N$ has the homotopy type of $(X, *)$, there is a map $g$ : $Y \rightarrow G(X)$ such that the pullback of $M$ by $g$ is fiber homotopically equivalent to $N$ as a $Y$-sectioned space. Moreover, $g$ is unique up to homotopy.

Then if $h: B \rightarrow G\left(E\left(S^{\prime}(F)\right)^{t}\right)$ is the classifying map for $E_{B}\left(S_{B}^{\prime}(T)\right)^{t}, T$ $\rightarrow B$ is orientable with respect to $E$ through dimension $t$ if and only if $h$ is nullhomotopic. If $F=S^{n}$ and $E=K(\mathrm{Z})$, then by Siegel [17] $G\left(E\left(S^{\prime}(F)\right)^{t}\right)$ $=K\left(\mathrm{Z}_{2}, 1\right)$ and $h$ is just the first SW class of the sphere fibration $T \rightarrow B$.

8. Appendix: Weak pair fibrations. In this section we give a brief exposition of weak pair fibrations. The results are mostly obvious generalizations of results of Dold [6] about weak fibrations. 
Let $B_{0}$ be a subspace of $B$. A continuous map $p:\left(T, T_{0}\right) \rightarrow\left(B, B_{0}\right)$ is a pair over $\left(B, B_{0}\right)$. If $p:\left(T, T_{0}\right) \rightarrow\left(B, B_{0}\right)$ and $p^{\prime}:\left(T^{\prime}, T_{0}^{\prime}\right) \rightarrow\left(B, B_{0}\right)$ are pairs over $\left(B, B_{0}\right)$, then a continuous map $\alpha:\left(T, T_{0}\right) \rightarrow\left(T^{\prime}, T_{0}^{\prime}\right)$ is a map over $\left(B, B_{0}\right)$ if $p^{\prime} \alpha=p$. A $B$-sectioned space $W$ can be though of as a pair $(W, B) \rightarrow(B, B)$ over $(B, B)$; then every map in $T B$ becomes a map over $(B, B)$.

$p:\left(T, T_{0}\right) \rightarrow\left(B, B_{0}\right)$ is said to be a trivial pair over $\left(B, B_{0}\right)$ if it is homeomorphic as a pair over $\left(B, B_{0}\right)$ to the projection $\left(B \times F, B_{0} \times F_{0}\right)$ $\rightarrow\left(B, B_{0}\right)$ for some $\left(F, F_{0}\right)$.

$p:\left(T, T_{0}\right) \rightarrow\left(B, B_{0}\right)$ and $p^{\prime}:\left(T^{\prime}, T_{0}^{\prime}\right) \rightarrow\left(B, B_{0}\right)$ are fiber homotopically equivalent as pairs over $\left(B, B_{0}\right)$ if there are maps $\alpha:\left(T, T_{0}\right) \rightarrow\left(T^{\prime}, T_{0}^{\prime}\right)$ and $\beta:\left(T^{\prime}, T_{0}^{\prime}\right) \rightarrow\left(T, T_{0}\right)$ over $\left(B, B_{0}\right)$ such that $\alpha \beta$ and $\beta \alpha$ are homotopic to $1_{\left(T^{\prime}, T_{0}^{\prime}\right)}$ and $1_{\left(T, T_{0}\right)}$ respectively, through maps over $\left(B, B_{0}\right)$.

$p:\left(T, T_{0}\right) \rightarrow\left(B, B_{0}\right)$ is a pair fibration if for every map $f:\left(Y, Y_{0}\right) \rightarrow$ $\left(T, T_{0}\right)$ and every homotopy $h:\left(Y, Y_{0}\right) \times I \rightarrow\left(B, B_{0}\right)$ such that $h(y, 0)=$ $p f(y)$ for $y \in Y$, there exists a homotopy $\bar{h}:\left(Y, Y_{0}\right) \times I \rightarrow\left(T, T_{0}\right)$ such that $\bar{h}(y, 0)=f(y)$ for $y \in Y$ and $p \bar{h}=h . p$ is a weak pair fibration if $\bar{h}$ exists whenever $h$ satisfies the additional condition: $h(y, t)=h(y, 0)$ for $y \in Y, 0 \leqslant t \leqslant 1 / 2$.

It is easy to see that if $p$ and $p^{\prime}$ are fiber homotopically equivalent pairs over $\left(B, B_{0}\right)$ and $p$ is a weak pair fibration, then $p^{\prime}$ is also a weak pair fibration. In particular, if $W \in T B$ and $W^{\prime} \in T B$ are fiber homotopically equivalent as $B$-sectioned spaces and $W \in T B$ (fib), then $W^{\prime} \in T B$ (fib) also.

The following four properties of weak pair fibrations (as well as many others) can be established in the same manner as the corresponding properties of weak fibrations. (See Dold [6] and Proposition (12) of Stasheff [18]. It is only necessary to check that the arguments given in [6] and [18] work in the category of pairs.)

PROPERTY 8.1. Let $p:\left(T, T_{0}\right) \rightarrow\left(B, B_{0}\right)$ and $p^{\prime}:\left(T^{\prime}, T_{0}^{\prime}\right) \rightarrow\left(B, B_{0}\right)$ be weak pair fibrations. Then a map $\alpha:\left(T, T_{0}\right) \rightarrow\left(T^{\prime}, T_{0}^{\prime}\right)$ over $\left(B, B_{0}\right)$ is a fiber homotopy equivalence of pairs over $\left(B, B_{0}\right)$ if and only if it is an ordinary homotopy equivalence of pairs.

Property 8.2. Assume $B$ admits a numerable covering $\left\{V_{\lambda}\right\}_{\lambda \in \Lambda}$ such that the inclusion $\left(V_{\lambda}, V_{\lambda} \cap B_{0}\right) \rightarrow\left(B, B_{0}\right)$ is nullhomotopic for every $\lambda$ and let $p:\left(T, T_{0}\right) \rightarrow\left(B, B_{0}\right)$ and $p^{\prime}:\left(T^{\prime}, T_{0}^{\prime}\right) \rightarrow\left(B, B_{0}\right)$ be weak pair fibrations. Then a map $\alpha:\left(T, T_{0}\right) \rightarrow\left(T^{\prime}, T_{0}^{\prime}\right)$ over $\left(B, B_{0}\right)$ is a fiber homotopy equivalence of pairs over $\left(B, B_{0}\right)$ if and only if the restriction of $\alpha$ to every fiber pair $\alpha_{b}:\left(p^{-1}(b), p^{-1}(b) \cap T_{0}\right) \rightarrow\left(p^{\prime-1}(b), p^{\prime-1}(b) \cap T_{0}^{\prime}\right)$ is an ordinary homotopy equivalence of pairs. 
Property 8.3. Let $B$ be as in 8.2. Then $p:\left(T, T_{0}\right) \rightarrow\left(B, B_{0}\right)$ is a weak pair fibration if and only if the restriction of $p$ to each pair $\left(p^{-1}\left(V_{\lambda}\right)\right.$, $\left.p^{-1}\left(V_{\lambda}\right) \cap T_{0}\right)$ is fiber homotopically equivalent as a pair over $\left(V_{\lambda}, V_{\lambda} \cap B_{0}\right)$ to a trivial pair over $\left(V_{\lambda}, V_{\lambda} \cap B_{0}\right)$.

Property 8.4. Let $p:\left(T, T_{0}\right) \rightarrow\left(B, B_{0}\right)$ be a weak pair fibration and assume $\left(B, B_{0}\right)$ has the homotopy type of a $\mathrm{CW}$ pair. Then $\left(T, T_{0}\right)$ has the homotopy type of a CW pair if and only if $\left(p^{-1}(b), p^{-1}(b) \cap T_{0}\right)$ has the homotopy type of a $\mathrm{CW}$ pair for each $b \in B$.

We now consider some constructions which produce weak pair fibrations. If $p: T \rightarrow B$ and $p^{\prime}: T^{\prime} \rightarrow B$ are spaces over $B$, we can form the "fiberwise" join $\bar{p}:\left(T *_{B} T^{\prime}, T \cup T^{\prime}\right) \rightarrow(B, B)$ as follows: Let $T \times_{B} T^{\prime}$ be the subspace of $T \times T^{\prime}$ consisting of $\left(x, x^{\prime}\right) \in T \times T^{\prime}$ satisfying $p(x)=p^{\prime}\left(x^{\prime}\right)$. $T *_{B} T^{\prime}$ is the quotient space obtained from $T \times{ }_{B} T^{\prime} \times I$ by identifying $\left(x_{0}, x_{0}^{\prime}, t_{0}\right) \in T \times_{B} T^{\prime} \times I$ with $\left(x_{1}, x_{1}^{\prime}, t_{1}\right) \in T \times_{B} T^{\prime} \times I$ if

$$
t_{0}=t_{1}=0, x_{0}=x_{1},
$$

or

$$
t_{0}=t_{1}=1, \quad x_{0}^{\prime}=x_{1}^{\prime} .
$$

We give $T *_{B} T^{\prime}$ the quotient topology and identify $T$ and $T^{\prime}$ with subspaces of $T *_{B} T^{\prime}$ in the obvious way. $\bar{p}$ is defined by $\bar{p}\left[x, x^{\prime}, t\right]=p(x)$.

THEOREM 8.5. If $p: T \rightarrow B$ and $p^{\prime}: T^{\prime} \rightarrow B$ are weak fibrations, then $\bar{p}:\left(T *_{B} T^{\prime}, T \cup T^{\prime}\right) \rightarrow(B, B)$ is a weak pair fibration.

PROof. Since every weak fibration is fiber homotopically equivalent to a fibration, we may assume that $p$ and $p^{\prime}$ are fibrations. Let $T \bar{*}_{B} T^{\prime}$ have the same point set as $T *_{B} T^{\prime}$ but the strong topology. (See Hall [8] for the definition of the strong topology.) Relabel $\bar{p}$ as $\tilde{p}$ when it is regarded as a map $\left(T \bar{*}_{B} T^{\prime}, T \cup T^{\prime}\right) \rightarrow(B, B)$. It is not difficult to see that the identity $\alpha:\left(T *_{B} T^{\prime}, T \cup T^{\prime}\right) \rightarrow\left(T \bar{*}_{B} T^{\prime}, T \cup T^{\prime}\right)$ is a fiber homotopy equivalence of pairs over $(B, B)$. In fact, we can construct an inverse as follows. Define $u: I \rightarrow I$ by

$$
\begin{aligned}
& u(t)=0 \quad \text { for } t \leqslant 1 / 4, \\
& =2 t-1 / 2 \text { for } 1 / 4 \leqslant t \leqslant 3 / 4 \text {, } \\
& =1 \text { for } t \geqslant 3 / 4 \text {. }
\end{aligned}
$$

Then $1 \times u: T \times T^{\prime} \times I \rightarrow T \times T^{\prime} \times I$ induces a map $\beta:\left(T \bar{*}_{B} T^{\prime}, T \cup T^{\prime}\right)$ $\rightarrow\left(T *_{B} T^{\prime}, T \cup T^{\prime}\right)$ over $(B, B)$. We leave it to the reader to verify that $\alpha$ and $\beta$ are fiber homotopy inverses of each other.

But Hall [8] has shown that $\tilde{p}$ is a pair fibration; hence $\bar{p}$ is a weak pair 
fibration. (In [8], Hall states only that the projection $T \bar{*}_{B} T^{\prime} \rightarrow B$ is a fibration, but his proof actually establishes that $\tilde{p}$ is a pair fibration.)

Corollary 8.6. If $T \rightarrow B$ is a weak fibration, then $S_{B}^{\prime}(T) \in T B$ (fib).

Proof. If $T^{\prime} \rightarrow B$ is the trivial double cover over $B$, then $S_{B}^{\prime}(T)=$ $T *_{B} T^{\prime}$.

THeOREM 8.7. Assume $B$ is locally compact and has the homotopy type of a $C W$ complex. Then if $W \in T B$ (fib), $E_{B}(W) \in T B$ (fib).

Proof. Since $B$ has the homotopy type of a $\mathrm{CW}$ complex, it admits a numerable open covering $\left\{V_{\lambda}\right\}_{\lambda \in \Lambda}$ such that the inclusion $V_{\lambda} \rightarrow B$ is nullhomotopic for each $\lambda$. Let $p:(W, B) \rightarrow(B, B)$ be the projection. By 8.3, $\left(p^{-1}\left(V_{\lambda}\right), V_{\lambda}\right)$ is fiber homotopically equivalent as a pair over $\left(V_{\lambda}, V_{\lambda}\right)$ to the projection $\left(V_{\lambda} \times F, V_{\lambda} \times *\right) \rightarrow\left(V_{\lambda}, V_{\lambda}\right)$ for some pointed space $F$.

Then $\left(E_{V_{\lambda}}\left(p^{-1}\left(V_{\lambda}\right)\right), V_{\lambda}\right)$ is fiber homotopically equivalent as a pair over $\left(V_{\lambda}, V_{\lambda}\right)$ to $\left(E_{V_{\lambda}}\left(V_{\lambda} \times F\right), V_{\lambda}\right)$. But since $V_{\lambda}$ is locally compact, $E_{V_{\lambda}}\left(V_{\lambda} \times F\right)=V_{\lambda} \times E(F)$ as a $V_{\lambda}$-sectioned space. Applying 8.3 again, we see that the projection $\left(E_{B}(W), B\right) \rightarrow(B, B)$ is a weak pair fibration.

REMARK. If $B$ is not assumed to be locally compact but $(W, B)$ is assumed to have the homotopy type of a $\mathrm{CW}$ pair, it can be shown that $\Omega_{B}^{m}\left(W \wedge_{B} E_{m}\right) \in T B$ (fib) if $W \in T B$ (fib).

\section{REFERENCES}

1. J. F. Adams, Stable homotopy and generalized homology, Mathematics Lecture Notes, University of Chicago, 1971.

2. D. W. Anderson, Resolutions of fibrations, Bol. Soc. Mat. Mexicana (2) 16 (1971), 26-28.

3. J. C. Becker, Cohomology and the classification of liftings, Trans. Amer. Math. Soc. 133 (1968), 447-475. MR 38 \#5217.

4. Extensions of cohomology theories, Illinois J. Math. 14 (1970), 551 584. MR $42 \# 8476$.

5. A. K. Bousfield and D. M. Kan, The homotopy spectral sequence of a space with coefficients in a ring, Topology 11 (1972), 79-106. MR 44 \#1031.

6. A. Dold, Partitions of unity in the theory of fibrations, Ann. of Math. (2) 78 (1963), 223-255. MR 27 \#5264.

7. S. Gitler and M. Mahowald, The geometric dimension of stable real vector bundles, Bol. Soc. Mat. Mexicana (2) 11 (1966), 85-107. MR 37 \#6922.

8. I. M. Hall, The generalized Whitney sum, Quart. J. Math. Oxford Ser. (2) 16 (1965), 360-384. MR 32 \#4698.

9. L. L. Larmore, Twisted cohomology theories and the single obstruction to lifting, Pacific J. Math. 41 (1972), 755-769.

10. M. Mahowald, On obstruction theory in orientable fiber bundles, Trans. Amer. Soc. 110 (1964), 315-349. MR 28 \#620.

11. W. S. Massey and F. P. Peterson, The cohomology structure of certain fiber spaces. I, Topology 4 (1964), 47-65. MR 32 \#6459.

12. J. F. McClendon, $A$ spectral sequence for classifying liftings in fiber spaces, Bull. Amer. Math. Soc. 74 (1968), 982-984. 
13. J. F. McClendon, Higher order twisted cohomology operations, Invent. Math. 7 (1969), 183-214. MR 40 \#3541.

14. - Obstruction theory in fiber spaces, Math. Z. 120 (1971), 1-17.

MR 45 \#6002.

15. J.-P. Meyer, Relative stable cohomotopy, Conference on Algebraic Topology, University of Illinois at Chicago Circle, 1968, pp. 206-212.

16. R. E. Mosher and M. C. Tangora, Cohomology operations and applications in homotopy theory, Harper \& Row, New York and London, 1968. MR 37 \#2223.

17. J. Siegel, $k$-invariants in local coefficient theory, Proc. Amer. Math. Soc. 29 (1971), 169-174. MR 46 \#6344.

18. J. D. Stasheff, A classification theorem for fibre spaces, Topology 2 (1963), 239-246. MR 27 \#235.

DEPARTMENT OF MATHEMATICS, NORTHWESTERN UNIVERSITY, EVANSTON, ILLINOIS 60201 (Current address of Mark Mahowald)

Current address (Robert Rigdon): Department of Mathematics, California State College at Dominguez Hills, Dominguez Hills, California 90747 